\title{
ESFUERZO DE MUESTREO PARA LA EVALUACIÓN DE LA DIVERSIDAD COLECTADA EN PIT- FALL EN LA RESERVA NACIONAL DE LACHAY - PERÚ.
}

David Ramírez C. ${ }^{1}$, Dante H. Pérez S. ${ }^{2}$ Edgar Sánchez I. ${ }^{1}$ y Germán Arellano C. ${ }^{2}$

\section{Resumen}

El trabajo se realizó en la Reserva Nacional de Lachay, Lima - Perú, el mes de febrero de 1998, durante el Fenómeno El Niño. Se determinó el tamaño mínimo de unidad muestral (TUM) para la evaluación de la diversidad en pit-fall, quedando estandarizado éste en dos trampas pit-fall, con un esfuerzo de siete días por cada trampa. Se obtuvieron, también, valores altos y significativos de correlación utilizando el índice de Pearson, entre la diversidad de sólo el orden Coleoptera y la diversidad de cuatro ordenes de insectos juntos (Hemiptera, Coleoptera, Hymenoptera y Homoptera). Los resultados sugieren que la diversidad de los Coleópteros puede ser considerada como representativa de la diversidad de la comunidad de insectos del suelo.

Palabras Claves: Diversidad, muestreo, insectos, Coleoptera, Lachay.

\begin{abstract}
The investigation was conducted at Lachay National Reserve, Lima - Peru, in the month of February 1998, during an El Niño Phenomenon. In order to evaluate soil entomological fauna the smallest sample size (SSS) was estimated. It was established at 2 pit-fall traps in an effort of seven days per trap. With the Pearson index high and significant correlation values were found between the order Coleoptera and the other four orders of insects (Hemiptera, Coleoptera, Hymenoptera and Homoptera). Thus, our results suggest that Coleoptera diversity can be established as representative of the diversity of the soil insect community.
\end{abstract}

Key Words: Diversity, sampling, insects, Coleoptera, Lachay.

\section{Introducción}

La preocupación ligada a la idea del "cuanto medir" en los estudios en ecología, fue enfocada inicialmente por la escuela fitosociológica con la idea del área mínima. Esta idea se refiere al tamaño requerido por la muestra para que sea representada adecuadamente la composición de especies de una comunidad (Ershaw, 1973). El área mínima a su vez se representa en una figura en cuyas ordenadas se encuentra el número de especies y en las abscisas el área muestreada. Las curvas generadas son las comúnmente llamadas curvas especies - área. Así, el área mínima está relacionada con la superficie en la cual esta curva comienza a ser horizontal (BraunBlanquet, 1979).

La idea de relacionar el número de especies con el área muestreada ha venido siendo tomada en cuenta no sólo en el estudio de la vegetación, sino, incorporada también con algunas modificaciones en los estudios de fauna. Dentro de los métodos derivados y empleados para este fin se encuentran principalmente: - la lista de las veinte especies (Mc
Kinnon \& Phillips, 1993), - el análisis del comportamiento del número de especies acumuladas versus la intensidad del esfuerzo de muestreo (ejemplo: Dallmeier \& Alonso, 1997) y - la evaluación del número de especies versus el número de individuos en las muestras; éste último método recomendado por Wolda (1983) para el estudio de la entomofauna, el cual indica mejor la riqueza de la misma que cualquier índice de diversidad.

Para Huston (1994), las diferencias en las curvas especies - área en las investigaciones comparativas de comunidades pueden sugerir también diferencias en los procesos ecológicos que existen en las mismas, aunque es la heterogeneidad espacial el principal condicionamiento de la forma de la curva. Sin embargo, Del Valle (1998), menciona que las curvas especies - área convencionales sufren principalmente de dos inconvenientes: el primero, es que en la mayoría de los grupos vivos evaluados estas curvas no alcanzan su valor asintótico, es decir tienden siempre a crecer, y el segundo, es el patrón gregario que predomina en las especies, lo que hace que si

${ }^{1}$ Laboratorio de Ecología de Procesos. Departamento Académico de Biología, Facultad de Ciencias de la Universidad Nacional Agraria la Molina. Lima Perú Apartado Postal 456 Lima 100 Perú. Correo electrónico: darc@lamolina.edu.pe / esi@lamolina.edu.pe

${ }^{2}$ Laboratorio de Control Biológico y Ecología de Artrópodos. Departamento Académico de Biología, Facultad de Ciencias de la Universidad Nacional Agraria La Molina Lima - Perú. Apartado postal 456 Lima 100 Perú. Correo electrónico: dahpes@lamolina.edu.pe acg@lamolina.edu.pe 
cambiamos el orden en el proceso de acumulación de muestras obtendremos curvas de diferentes formas. Es por ello que como complemento del análisis de las curvas de especies - área asociado a la idea de diversidad de especies, Margalef (1991) propone la noción de Espectro de Diversidad (variación del índice de diversidad en función del tamaño de la muestra) como un complemento y ampliación al entendimiento de la organización espacial y complejidad de las comunidades.

Para la evaluación de la entomofauna, son muy pocos los trabajos en los que se analizan los aspectos mencionados. Podemos referir el trabajo de Eglenton et al. (1995) con termitas, en donde se muestran curvas especies-área de estos insectos, en lugares con diferentes grados de perturbación en un bosque húmedo tropical en Camerún.

Este trabajo por tanto pretende: 1.- Cubrir información respecto a los tamaños de unidad muestral mínimos para la evaluación de la diversidad de especies colectadas en pit-fall; 2.- Justificar el monitoreo de sólo un orden como representativo de la diversidad total de la misma en las Lomas de Lachay, siendo la única información entomológica publicada las listas de las principales especies de Aguilar (1964, 1975, 1976 y 1985) para los ecosistemas de Lomas Costeras de nuestro país, y el trabajo de Saito (1976), en la Reserva Nacional de Lachay específicamente.

Lugar de Estudio

El trabajo se realizó en la Reserva Nacional

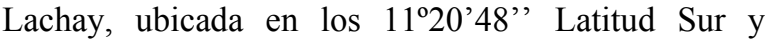
77²19'45' Longitud Oeste, a $105 \mathrm{Km}$. al norte de la ciudad de Lima, en las provincias de Chancay y Huaral, con una superficie de $5070 \mathrm{Ha}$.

Las Lomas de Lachay presentan dos épocas marcadas determinadas por la disponibilidad de agua proveniente de la neblina que condensa y precipita. Así, la época seca abarca los meses de enero a marzo donde se registra el mínimo valor de la precipitación mensual (promedio para el mes de febrero igual a 1.13 mm.), y la evaporación y la temperatura expresan los mayores valores del año $(108.0 \mathrm{~mm}$ de evaporación promedio para el mes de marzo y $21.8^{\circ} \mathrm{C}$ en el mes de febrero). La época húmeda se inicia entre mayo y julio, y concluye entre setiembre y noviembre. El valor máximo de la precipitación mensual $(31.3 \mathrm{~mm}$.) y los valores mínimos de evaporación $(11.9 \mathrm{~mm}$. en promedio) y temperatura $\left(13.3^{\circ} \mathrm{C}\right)$ se dan en agosto (Ordoñez y Faustino, 1983). La estacionalidad marcada de las variables meteorológicas mencionadas condicionan el ciclo de presencia o ausencia de vegetación principalmente herbácea en las lomas durante el año.

La colección de información sobre la composición biológica de la comunidad de insectos se realizó en el mes de febrero de 1998, en pleno desarrollo del evento del Niño. Por ello durante este período las lomas costeras reverdecieron debido a la presencia inusual de neblina.

\section{Materiales y métodos}

El análisis de esta información, que incluye la identificación de especímenes y el posterior tratamiento estadístico de los datos, se desarrollaron en el Laboratorio de Control Biológico y el Laboratorio de Ecología de Procesos del Departamento de Biología de la Universidad Nacional Agraria La Molina.

El muestreo fue realizado en treinticuatro estaciones ubicadas sistemáticamente en la reserva. Para la colecta de insectos se utilizaron trampas pitfall (Ausden, 1996), colocándose tres trampas en treintiuno estaciones y cuatro en tres, cada una con un esfuerzo total de siete días.

Las muestras colectadas en el campo fueron llevadas al laboratorio, donde cada muestra fue filtrada utilizando tamices de diferente diámetro de abertura, separándose de esta manera los insectos capturados. Luego se eligieron al azar las trampas de cinco estaciones, de donde se codificaron y cuantificaron las especies y/o fenotipos adultos de cuatro ordenes de insectos (Hemiptera, Coleoptera, Hymenoptera y Homoptera). Se realizó la cuantificación de los mismos para la determinación de la abundancia de cada especie en cada trampa, con cuyos datos se determinó la diversidad de especies de los cuatro ordenes de insectos de manera conjunta y del orden Coleoptera de manera independiente, utilizando el índice de diversidad de Shannon-Weaver - $\mathrm{H}^{\prime}$ (Margalef 1991),

$$
\mathrm{H}^{\prime}=-\mathrm{pi} \log _{2} \mathrm{pi}
$$

donde:

- $\quad \mathrm{pi}=$ proporción de individuos de la especie $\mathrm{i}$ en la muestra $=\mathrm{ni} / \mathrm{N}$

- $\quad$ ni $=$ número de individuos de la especie $\mathrm{i}$ en la muestra

- $\quad \mathrm{N}=$ número total de individuos en la muestra.

Para la determinación del tamaño mínimo de unidad muestral (TUM) en la evaluación de la diversidad de la entomofauna, se analizó las curvas del número de especies acumuladas vs. el incremento de esfuerzo de muestreo y también los espectros de diversidad, utilizando el índice de Shannon-Weaver, graficando cada valor acumulado por TUM del índice con su respectivo intervalo de confianza (IC) generado a partir de la obtención de la variancia (Var $\left.\mathrm{H}^{\prime}\right)$ del mismo (Magurran 1988):

$$
\mathrm{VarH}=\frac{\sum \text { pi }(\operatorname{logpi})^{2}-\left(\sum \text { pilogpi }\right)^{2}}{\mathrm{~N}}-\frac{\mathrm{S}-1}{2 \mathrm{~N}^{2}}
$$

donde :

- $\mathrm{S}=$ Número total de especies en la muestra 


$$
\text { I.C. }=\sqrt{\frac{\operatorname{VarH}^{\prime}}{N}} \times t(N-1)
$$

donde:
- $\quad \mathrm{t}(\mathrm{N}-1)=$ Valor de la prueba $\mathrm{t}-$ Student con $\mathrm{N}-1$ grados de libertad

El análisis se realizó para los cuatro ordenes de insectos de manera conjunta (Hemiptera, Coleoptera, Hymenoptera y Homoptera) y para el orden Coleoptera independientemente, en las cinco estaciones de muestreo elegidas al azar.

Para el establecimiento de la representatividad del orden Coleoptera como significativa del comportamiento de la diversidad de la comunidad de insectos, se diseñaron diagramas de dispersión de la diversidad de los cuatro órdenes de insectos vs. la diversidad del orden Coleoptera, para la determinación de la correlación existente, mediante el uso del índice de Pearson, y su respectiva significancia estadística (Steel y Torrie, 1988).

La colección de referencia de los cuatro ordenes de insectos fueron depositados en el Museo de Entomología de la Facultad de Agronomía de la Universidad Nacional Agraria La Molina. El trabajar con cuatro órdenes de insectos, demandaría mucho esfuerzo y tiempo, por lo cual se quiso comprobar si trabajando solamente con el orden Coleoptera se puede obtener una representatividad significativa del comportamiento de la diversidad de la comunidad de insectos

\section{Resultados y discusión}

Determinación del tamaño mínimo de unidad muestral (TUM):

En la información obtenida para la determinación del tamaño mínimo de unidad muestral (TUM) se puede observar que las curvas número de especies acumuladas vs. el incremento de esfuerzo de muestreo, en general, aumentan su valor con el incremento del TUM, lo cual coincide con el patrón observado por Del Valle (1998). Sin embargo, en los espectros de diversidad de las estaciones evaluadas (excepto la $\mathrm{N}^{\circ}$ 5), el valor del índice de diversidad acumulado para el TUM 2 y TUM 3, presenta valores críticos en el intervalo de confianza muy cercanos (Figuras: 1.a y 1.b de la estación 1, 2.a y 2.b de la estación 2, 3.a y 3.b de la estación 3, 4.a y 4.b de la estación 4, 5.a y 5.b de la estación 5), siendo la diferencia entre estos menor o igual a 0.16 bits/individuo para los cuatro ordenes juntos (Hemiptera, Coleoptera, Hymenoptera y Homoptera) y menor o igual a 0.11 bits/individuo para el orden Coleoptera, e inclusive en las estaciones 1 y 4 se puede observar un traslape (Figuras 1.b y 4.b), lo que nos estaría indicando que no existe diferencia significativa en la diversidad de especies entre el TUM 2 y TUM 3. De lo mencionado, para la evaluación de la diversidad de especies colectadas en pit-fall, se recomienda un TUM de dos trampas pit-fall con un esfuerzo de 7 días cada una.

Elección del Orden Coleoptera para el análisis de diversidad.

Los diagramas de dispersión se muestran en las Figuras $6-10$. Como se puede observar en cuatro de las cinco estaciones la correlación de la diversidad acumulada por TUM, entre los cuatro ordenes juntos de insectos y el orden Coleoptera, es positiva (Tablas $1-5)$. Si bien se obtuvieron valores en el índice de Pearson mayores a 0.9, no se llegó a alcanzar significancia estadística en la mayoría de estaciones debido a la poca cantidad de datos para la correlación ( $\mathrm{n}=3$ para las estaciones $1-3$ y $\mathrm{n}=4$ para las estaciones 4 y 5), sin embargo para el caso de la estación 1 (Cuadro 1) se obtuvo un $\mathrm{p}<0.05$, lo que indica una correlación altamente significativa, y en las estaciones 2 y 5 (Tabla 1 y 5 ) se observa un $\mathrm{p}<0.1$. Estos valores de correlación nos estarían sugiriendo que la diversidad específica de los cuatro ordenes juntos evaluados en conjunto es reflejada por el comportamiento de la diversidad del orden Coleoptera en cada TUM.

A su vez, la importancia biológica de trabajar con los coleópteros radica en que dentro de este orden podemos encontrar dos grupos bien diferenciados según sus hábitos alimenticios: fitófagos $\mathrm{y}$ depredadores, los cuales podrían estar permitiéndonos establecer una relación directa con la dinámica de la vegetación y de la entomofauna del suelo, respectivamente.

\section{Conclusiones}

Las curvas, especies acumuladas versus esfuerzo de muestreo, tienden a aumentar en todas las estaciones evaluadas.

Se sugiere trabajar con un total de dos trampas pitfall con un esfuerzo de muestreo de 7 días cada una (es decir un TUM 2), para la evaluación de la diversidad de especies colectadas en pit-fall.

El orden Coleoptera muestra los requerimientos tanto estadísticos como biológicos para poder ser considerado como representativo de la diversidad de especies colectadas en pir-fall en las Lomas de Lachay.

\section{Litertura citada}

Aguilar P. 1964. Especies de artrópodos registrados en las Lomas de los alrededores de Lima. Rev. Per. Ent. 7 (1): 93 - 95.

1975. Nota sobre los Embioptera (Insecta) de la Regíon Desértico-Costera del Perú. Rev. Per. Ent. 18 (1): 127-128. 
1976. Fauna Desértico-Costera Peruana I: Invertebrados más frecuentes en las Lomas. Rev. Per. Ent. 19(1): 67 - 70.

1985. Fauna de las lomas costeras del Perú. Boletín de Lima. 41(7): 17 - 28.

Ausden M. 1996. Invertebrates. En : Sutherland, W. (edit.). Ecological census techniques a handbook Cambridge University Press.

Braun-Blanquet J. 1979. Fitosociología, bases para el estudio de las comunidades vegetales. H. Blume Ediciones, Madrid.

Dallmeier F. \& Alonso A. 1997. Biodiversity assessment and long-term monitoring of the Lower Urubamba Region in Peru. Phase II: San Martin-3 and Cashiriari-2 well sites. Executive Summary. Smithsonian Institution, Washington, D.C.

Del Valle J. 1998. Curva Especies Area y Riqueza Biológica: La asíntota de la curva especies-área como expresión de la riqueza biológica. http://www.icfes.gov.co/revistas/cronica/asintota.h tml. Noviembre.

Eglenton P., Bignel D., Watte W., Wood T. \& Lawton J. 1995. The species richness of termite (Isoptera) under differing levels of forest disturbance in the Hbalmayo Forest Reserve, southern Cameroon. Journal of Tropical Ecology. 11: 89 - 98 .

Ershaw K. 1973. Quantitative and dynamic plant ecology. Edward Arnold Publishers. London.

Huston H. 1994. Biological diversity, the coexistance of species on changing landascapes. Cambrigde University Press.

Margalef R. 1991. Ecología. Ediciones Omega S.A. $7^{\circ}$ edición, Barcelona.

Magurran A. 1988.Diversidad ecológica y su medición. Ediciones Vedr.

Mc Kinnon J. \& Phillips. 1993. A field guide to the birds of Borneo, Sumatra Java and Bali, Oxford. Oxford Univ. Press.

Ordoñez J. y Fautino, J. 1983. Evaluación del potencial hídrico en las Lomas Costeras del Perú (Lachay-Iguanil). Zonas Aridas $\mathrm{N}^{\circ} 3$. Lima.

Saito C. 1976. Bases para el establecimiento y manejo de una unidad de conservación en las Lomas de Lachay, Perú. Tesis para optar el título de Ingeniero Forestal. UNALM, Lima.

Steel R. y Torrie J. 1988. Bioestadística: Principios y Procedimientos. Segunda Edición. Mc Graw- Hill. Mexico.

Wolda H. 1983. Diversidad de la Entomofauna y cómo Medirla. Informe Final IX CLAZ Perú. : 181 186.

\section{ANEXOS: TABLAS Y FIGURAS}

\begin{tabular}{|c|c|c|}
\hline \multicolumn{3}{|c|}{$\begin{array}{l}\text { TABLA } 1 \text {.- ESTACION } 1 \\
\text { MATRIZ DE CORRELACIÓN DE PEARSON }\end{array}$} \\
\hline & Cuatro Ordenes & Orden Coleoptera \\
\hline Cuatro Ordenes & 1.000 & \\
\hline Orden Coleoptera & $1.000(0.180)$ & 1.000 \\
\hline
\end{tabular}

\begin{tabular}{|c|c|c|}
\hline \multicolumn{3}{|c|}{$\begin{array}{l}\text { TABLA } 2 \text {.- ESTACION } 2 \\
\text { MATRIZ DE CORRELACIÓN DE PEARSON }\end{array}$} \\
\hline & Cuatro Ordenes & Orden Coleoptera \\
\hline Cuatro Ordenes & 1.000 & \\
\hline Orden Coleoptera & $0.934(0.233)$ & 1.000 \\
\hline
\end{tabular}

\begin{tabular}{|c|c|c|}
\hline \multicolumn{3}{|c|}{$\begin{array}{l}\text { TABLA } 3 \text {-- ESTACION } 3 \\
\text { MATRIZ DE CORRELACIÓN DE PEARSON }\end{array}$} \\
\hline $\begin{array}{l}\text { Cuatro Ordenes } \\
\text { Orden Coleoptera }\end{array}$ & $\begin{array}{l}\text { Cuatro Ordenes } \\
1.000 \\
0.991(0.086)\end{array}$ & Orden Coleoptera \\
\hline \multicolumn{3}{|c|}{ Número de Observaciones : 3} \\
\hline
\end{tabular}

\begin{tabular}{|c|c|c|}
\hline \multicolumn{3}{|c|}{$\begin{array}{l}\text { TABLA } 4 \text {.- ESTACION } 4 \\
\text { MATRIZ DE CORRELACIÓN DE PEARSON }\end{array}$} \\
\hline $\begin{array}{l}\text { Cuatro Ordenes } \\
\text { Orden Coleoptera }\end{array}$ & $\begin{array}{l}\text { Cuatro Ordenes } \\
1.000 \\
0.230(0.770)\end{array}$ & Orden Coleoptera \\
\hline Número de Observ & aciones : 4 & \\
\hline
\end{tabular}

\begin{tabular}{|c|c|c|}
\hline \multicolumn{3}{|c|}{$\begin{array}{l}\text { TABLA } 5 \text {.- ESTACION } 5 \\
\text { MATRIZ DE CORRELACIÓN DE PEARSON }\end{array}$} \\
\hline $\begin{array}{l}\text { Cuatro Ordenes } \\
\text { Orden Coleoptera }\end{array}$ & $\begin{array}{l}\text { Cuatro Ordenes } \\
1.000 \\
0.945(0.055)\end{array}$ & Orden Coleoptera \\
\hline Número de Obser & aciones : 4 & \\
\hline
\end{tabular}



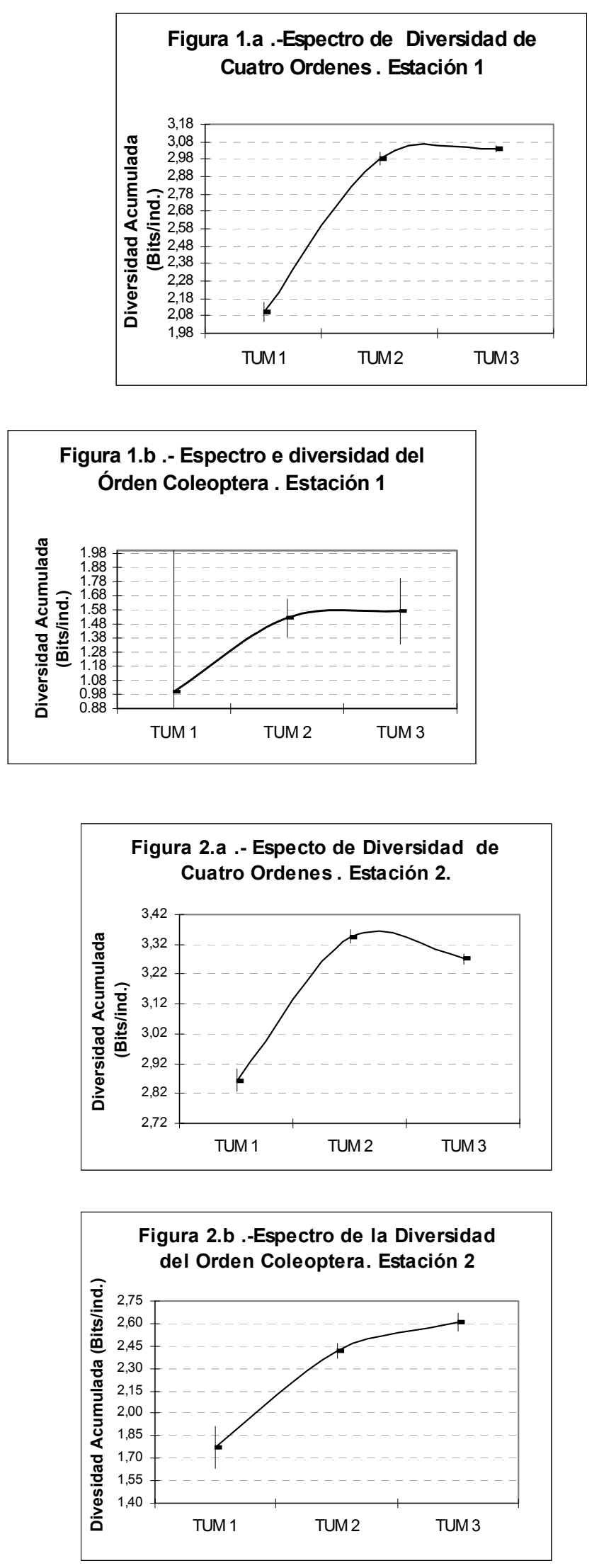

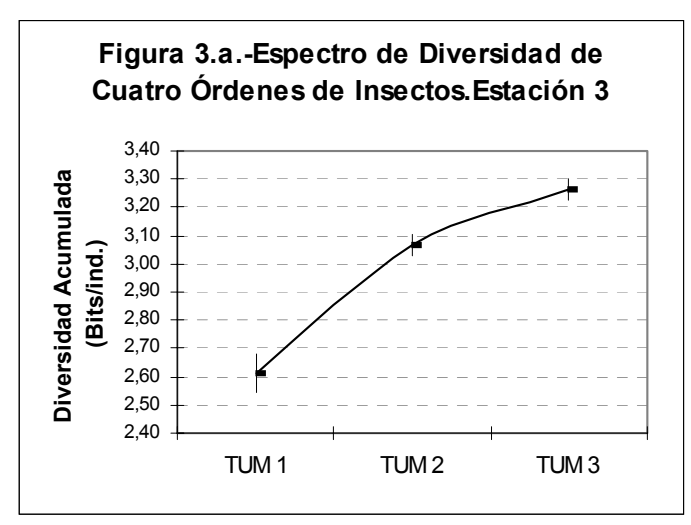

Figura 3.b .- Espetro de Diversidad del Órden Coleoptera . Estación 3.

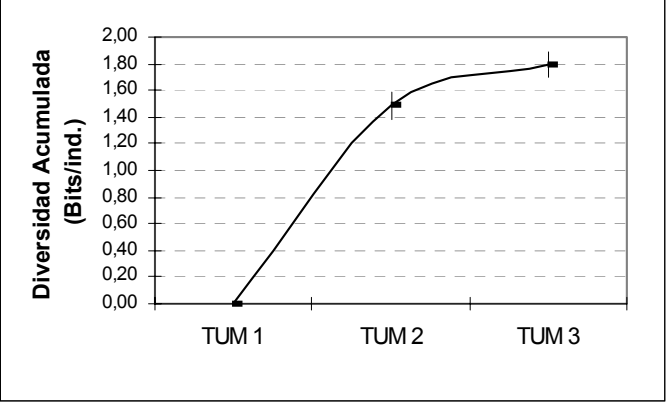

Figura 4.a .-Espectro de Diversidad de Cuatro Órdenes de Insectos. Estación 4
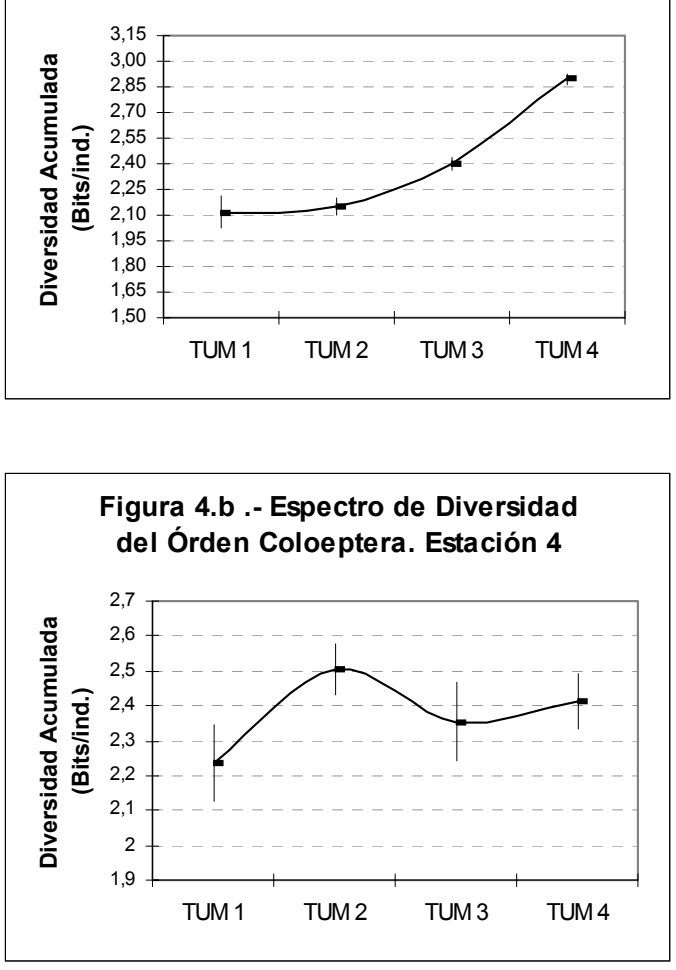


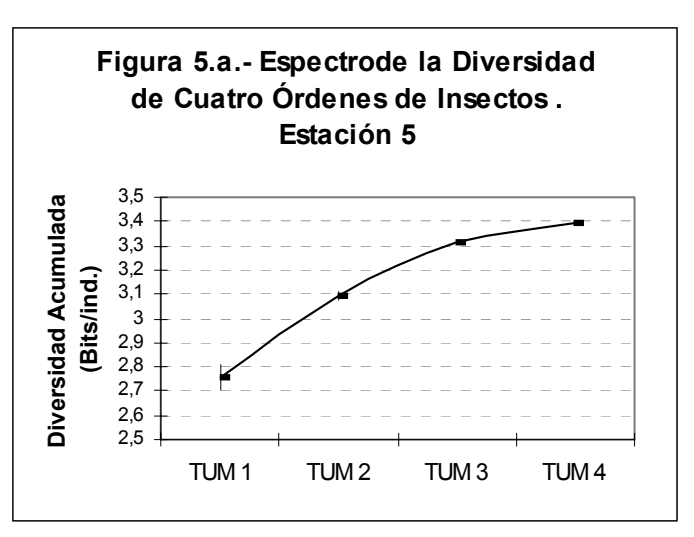

Figura 5.b.- Espectro de Diversidad del Órden Coleoptera . Estación 5

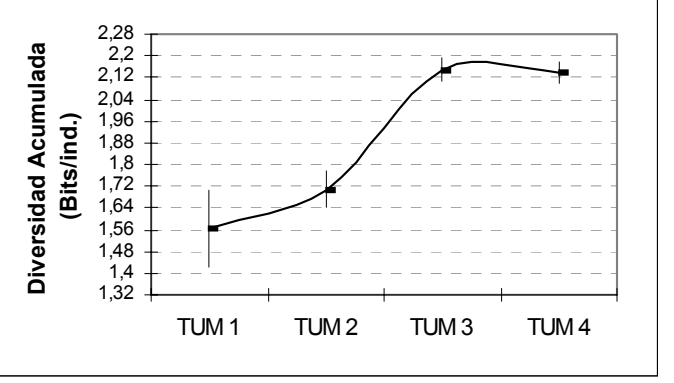

Figura 6.- Diagrama de dispersión de valores de diversidad acumulada. Estación 1

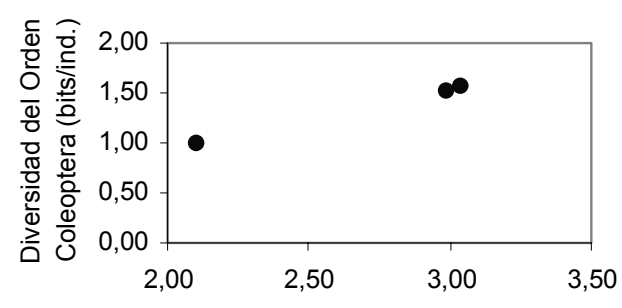

Diversidad de Cuatro Ordenes (bits/ind.)

Figura 7.- Diagrama de dispersión de valores de diversidad acumulada. Estación 2

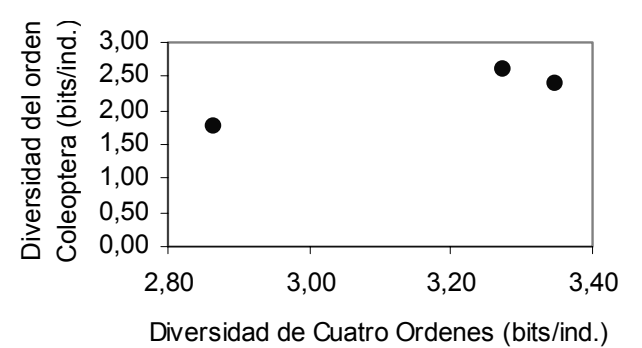

Diversidad de Cuatro Ordenes (bits/ind.)
Figura 8 .- Diagrama de dispersión de valores de diversidad acumulada . Estación 3

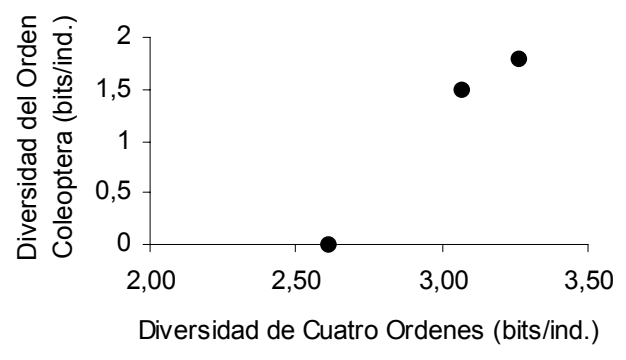

Figura 9.- Diagrama de dispersión de valores de diversidad acumulada .

Estación 4

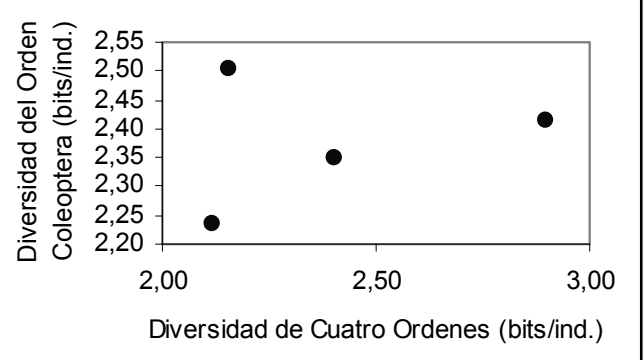

Figura 10.- Diagrama de dispersión de valores de diversidad acumulada . Estación 5

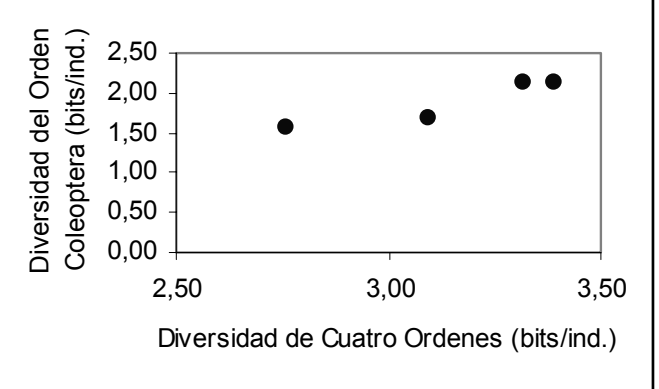

